This item was submitted to Loughborough's Research Repository by the author.

Items in Figshare are protected by copyright, with all rights reserved, unless otherwise indicated.

\title{
Alginate-lavender nanofibers with antibacterial and anti-inflammatory activity to effectively promote burn healing
}

\section{PLEASE CITE THE PUBLISHED VERSION}

http://dx.doi.org/10.1039/C5TB02174J

\section{PUBLISHER}

(c) Royal Society of Chemistry

\section{VERSION}

AM (Accepted Manuscript)

\section{PUBLISHER STATEMENT}

This work is made available according to the conditions of the Creative Commons Attribution-NonCommercialNoDerivatives 4.0 International (CC BY-NC-ND 4.0) licence. Full details of this licence are available at: https://creativecommons.org/licenses/by-nc-nd/4.0/

\section{LICENCE}

CC BY-NC-ND 4.0

\section{REPOSITORY RECORD}

Hajiali, Hadi, Maria Summa, Debora Russo, Andrea Armirotti, Virgilio Brunetti, Rosalia Bertorelli, Athanassia Athanassiou, and Elisa Mele. 2016. "Alginate-lavender Nanofibers with Antibacterial and Anti-inflammatory Activity to Effectively Promote Burn Healing". Loughborough University. https://hdl.handle.net/2134/20376. 


\section{Journal of}

Materials Chemistry B

\section{Accepted Manuscript}

This article can be cited before page numbers have been issued, to do this please use: H. Hajiali, M. Summa, D. Russo, A. Armirotti, V. Brunetti, R. Bertorelli, A. Athanassiou and E. Mele, J. Mater. Chem. B, 2016, DOI: 10.1039/C5TB02174J.

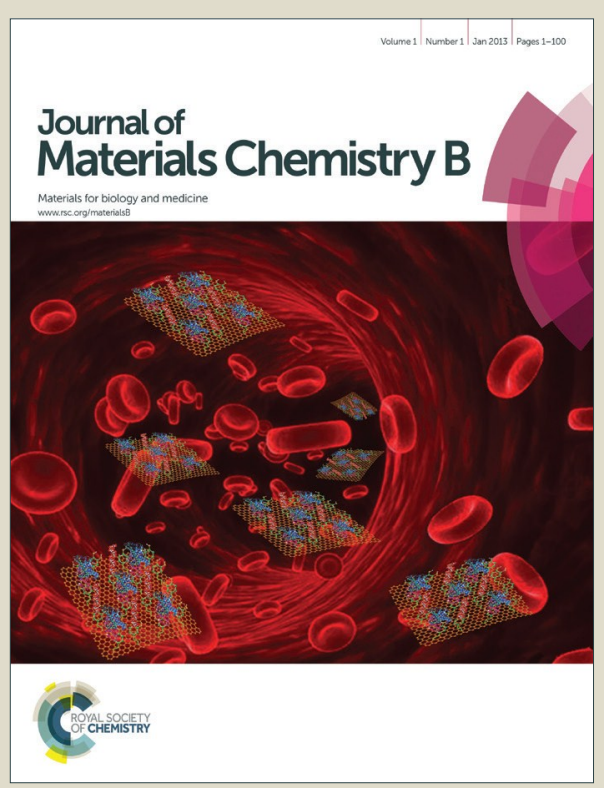

This is an Accepted Manuscript, which has been through the Royal Society of Chemistry peer review process and has been accepted for publication.

Accepted Manuscripts are published online shortly after acceptance, before technical editing, formatting and proof reading. Using this free service, authors can make their results available to the community, in citable form, before we publish the edited article. We will replace this Accepted Manuscript with the edited and formatted Advance Article as soon as it is available.

You can find more information about Accepted Manuscripts in the Information for Authors.

Please note that technical editing may introduce minor changes to the text and/or graphics, which may alter content. The journal's standard Terms \& Conditions and the Ethical guidelines still apply. In no event shall the Royal Society of Chemistry be held responsible for any errors or omissions in this Accepted Manuscript or any consequences arising from the use of any information it contains. 


\section{Alginate-lavender nanofibers with antibacterial and anti- inflammatory activity to effectively promote burn healing}

Received 00th January 20xx, Accepted 00th January 20xx

DOI: $10.1039 / \times 0 x \times 00000 x$

www.rsc.org/

\author{
Hadi Hajiali ${ }^{*}, a, b$, Maria Summa ${ }^{c}$, Debora Russo ${ }^{c}$, Andrea Armirotti ${ }^{c}$, Virgilio Brunetti ${ }^{d}$, Rosalia \\ Bertorelli ${ }^{\mathrm{c}}$, Athanassia Athanassiou ${ }^{*}$, , Elisa Mele ${ }^{*, a, e}$
}

One of the current challenges in wound care is the development of multifunctional dressings that can both protect the wound from external agents and promote the regeneration of the new tissue. Here, we show the combined use of two naturally derived compounds, sodium alginate and lavender essential oil, for the production of bioactive nanofibrous dressings by electrospinning, and their efficacy for the treatment of skin burns induced by midrange ultraviolet radiation (UVB). We demonstrate that the engineered dressings reduce the risk of microbial infection of the burn, since they stop the growth of Staphylococcus aureus. Furthermore, they are able to control and reduce the inflammatory response that is induced in human foreskin fibroblasts by lipopolysaccharides, and in rodents by UVB exposure. In particular, we report a remarkable reduction of pro-inflammatory cytokines when fibroblasts or animals are treated with the alginate-based nanofibers. The down-regulation of cytokines production and the absence of erythema on the skin of the treated animals confirm that the here described dressings are promising as advanced biomedical devices for burn management.

\section{Introduction}

An ideal biomedical device for wound care should promote the complete regeneration of the injured tissue, effectively restore its biological activity and aesthetic aspect, while reducing inflammation and preventing microbial invasion. ${ }^{1,2}$ Efforts for achieving this goal are leading to the replacement of traditional passive products with advanced ones. ${ }^{3}$ Among these, alginate-based dressings are attractive for their capability to release bioactive compounds and to maintain a moist environment around the wound, promoting tissue granulation and re-epithelialization..$^{4-8}$ Typically they are available in form of freeze-dried foams or non-woven microfibers, though great research interest is nowadays devoted towards nanofibrous matrices. In particular, nanofibers produced by electrostatic spinning have high

\footnotetext{
${ }^{a}$ Smart Materials, Istituto Italiano di Tecnologia, via Morego 30, 16163 Genoa, Italy.

${ }^{b}$ DIBRIS, University of Genoa, via Opera Pia 13, 16145, Genoa, Italy.

' PharmaChemistry, Drug Discovery and Development, Istituto Italiano di Tecnologia, Via Morego 30, 16163, Genoa, Italy.

${ }^{d}$ Center for Biomolecular Nanotechnologies, Istituto Italiano di Tecnologia @UniLe, Via Barsanti, 73010 Arnesano, Lecce, Italy.

${ }^{e}$ Current Address: Department of Materials, Loughborough University, Loughborough, LE11 3TU, United Kingdom.
}

\section{* Corresponding authors: \\ Hadi.Hajiali@iit.it \\ Athanassia.Athanassiou@iit.it \\ E.Mele2@lboro.ac.uk}

+ Footnotes relating to the title and/or authors should appear here.

Electronic Supplementary Information (ESI) available: [details of any supplementary information available should be included here]. See DOI: $10.1039 / x 0 \times x 00000 x$ potentiality in the wound healing field because their porosity promotes nutrient transport and gas permeation, their morphological organization mimics the native tissue, and their mechanical properties can be engineered..$^{5,11}$ The intrinsic high surface area of nanofibers is also attractive for the delivery of drugs and active agents. ${ }^{2,12}$

Alginate structures cross-linked with Calcium ions $\left(\mathrm{Ca}^{2+}\right)$ are mainly used for the treatment of highly exuding wounds and burns. $^{5,13,14}$ For instance, the haemostatic activity of dry Caalginate felts has been utilized for minimizing blood loss after the excision of deep burns to hands. ${ }^{15}$ It has been also demonstrated that films of this polysaccharide and chitosan in combination with laser therapy help the epithelization and vascularization of dermal burns. ${ }^{16}$ Furthermore, since burn wounds are highly exposed to the risk of microbial colonization that possibly determines local and systemic infections and delays the healing process, ${ }^{17,} 18$ alginate dressings containing silver $^{19,20}$ and other antimicrobial compounds ${ }^{21}$ have been developed. Despite the benefits offered by Ca-alginate based biomedical devices, studies in literature have pointed out that the release of $\mathrm{Ca}^{2+}$ ions from the dressing to the wound site stimulates the production of pro-inflammatory cytokines, such

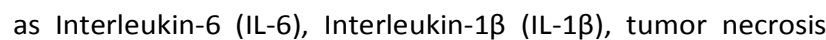
factor- $\alpha$ (TNF- $\alpha$ ), and the chemotactic cytokine IL- $8 .{ }^{22,23}$ The occurrence of this phenomenon, even if it is advantageous for other clinical situations, is discouraged in burn management, where the local reduction of the inflammation response is advocated. $^{24,25} \mathrm{IL}-6, \mathrm{IL}-1 \beta$, and TNF- $\alpha$ are, in fact, the most important cytokines involved in the inflammation phase of burn-induced skin damages. ${ }^{26}$ They are also detected after the acute skin exposure to midrange ultraviolet radiation (UVB), such as after sunburn. ${ }^{27}$ 
A plant extract that is traditionally used for its antiinflammatory and calming activity in case of minor burns and insect bites is Lavender essential oil (LO). This oil is derived from the flowers of Lavandula angustifolia through steam distillation and exhibits a variety of therapeutic effects: ${ }^{28-30}$ it reduces anxiety, acts as antioxidant, possesses anticancer and anti-mutagenic properties, relieves pain, and it is suitable for the management of central nervous system disorders. LO is composed by linalool and linalyl acetate, and by more than hundred other compounds. ${ }^{31}$ It has been demonstrated that its main components (linalool and linalyl acetate) are responsible for the anti-inflammatory activity. ${ }^{32,33}$ Moreover, LO possesses antibacterial and antifungal properties, ${ }^{28,34,35}$ pointing out its efficacy against Staphylococcus aureus that is the bacterium mostly responsible for burn wound infections. ${ }^{36}$ Electrospun nanofibers of Polyacrylonitrile (PAN) containing lavender oil have been recently developed for antibacterial and drug delivery applications. ${ }^{37}$ Although both inorganic and organic antiseptic agents have shown a certain degree of success for various medical applications, the use of all-natural medicinal extracts, such as LO, can further boost pharmaceutical applications and improve patient recovery and quality of life.

Here we show that dressings constituted by electrospun nanofibers of sodium alginate containing lavender essential oil are effective for the treatment of UVB-induced skin injuries. Invitro studies revealed that these entirely natural systems were highly biocompatible and able to inhibit the proliferation of $S$. aureus. Together with antibacterial activity, the produced alginate-based nanofibers expressed a remarkable antiinflammatory efficacy that was demonstrated in-vitro on lipopolysaccharide-stimulated human foreskin fibroblasts, and in-vivo on rodent model of UVB burns. In particular, a significant decrement of the production of pro-inflammatory cytokines was observed for both cells and animals. Interestingly, no marks of erythema were detected on the skin of the injured animals that were treated with the electrospun dressings, indicating that the treatment promptly stopped the inflammatory response. Differently from other topical preparations for the management of burn wounds, the here described biomedical devices perform dual functions (antibacterial and anti-inflammatory) and, thus, have potentialities to fill the void of multifunctional dressings that the market is still facing.

\section{Experimental}

\subsection{Materials and cells}

Sodium Alginate (SA), Polyethylene Oxide (PEO, $M_{W}=600,000$ g/mol), Pluronic F127, Dimethylformamide (DMF), Bacterriological agar, and Lysogeny broth (LB), Lipopolysaccharides (LPS) from Escherichia coli (serotype 026:B6), and dexamethasone (DXM) were purchased from Sigma Aldrich (St. Louis, MO, USA). Dulbecco's modified Eagle's medium (DMEM), fetal bovine serum (FBS), L-Glutamine and Penicillin-Streptomycin were from Euroclone (Milan, Italy). Lavender oil (LO) was obtained from Maitreya-Natura (Italy).
Staphylococcus aureus bacteria and human foreskin fibroblast (HFF-1) were from ATCC ${ }^{\circledR}$.

\subsection{Preparation of the alginate-based nanofibers by electrospinning}

Solutions for electrospinning were prepared by separately dissolving SA and PEO powders in distilled water at concentration of $4 \% \mathrm{w} / \mathrm{v}$. The SA and PEO solutions were mixed at a volume ratio of $8: 2$, and $5 \% \mathrm{v} / \mathrm{v}$ of DMF and $1.5 \%$ $\mathrm{w} / \mathrm{v}$ of Pluronic $\mathrm{F} 127$ were added and stirred overnight at room temperature. SA-PEO solution containing $5 \% \mathrm{v} / \mathrm{v}$ of Lavender oil was stirred in order to emulsify the oil in the water phase, before being electrospun. For the electrospinning process, a syringe with a stainless-steel 23-gauge needle was filled with the final solution (PEO, SA-PEO or SA-PEO/LO) and connected to a syringe pump (NE-1000, New Era Pump Systems, Inc.) working at a constant flow rate of $0.5 \mathrm{~mL} /$ hour. The needle was clamped to the positive electrode of a highvoltage power supply (EH4OR2.5, Glassman High Voltage, Inc.) generating $25 \mathrm{kV}$, and the ground electrode was connected to an aluminum collector (air gap distance of $20 \mathrm{~cm}$ ).

\subsection{Characterization of the nanofibrous dressings}

\section{Morphological investigations}

The morphology and size distribution of the electrospun nanofibers were analyzed by scanning electron microscopy (SEM). Prior to imaging, the fibrous mats were covered with 5 $\mathrm{nm}$ of gold, deposited by ion sputtering. The diameter distribution of the fibers was determined by processing the SEM images by Image J program.

\section{Chemical analysis}

The chemical analysis of the elecrospun mats (SA-PEO and SAPEO/LO) and of the pure essential oil was carried out by Raman spectroscopy, using a Horiba Jobin-Yvon $\mu$ Raman operating with a He-Ne laser source. The wavelength of the laser radiation was $632.8 \mathrm{~nm}$ and the objective used was a 50x with a slit aperture of about $200 \mu \mathrm{m}$.

\section{Wetting studies}

In order to evaluate the wettability of the nanofibers, the water contact angle (WCA) was measured by a video-based optical contact angle measuring instrument DataPhysics OCA 20 (Germany). Water droplets of a volume of $10 \mu \mathrm{l}$ were gently placed on the surfaces of the samples and the measurements were conducted on three different sample areas and averaged for each sample.

\section{Mechanical tests}

The mechanical properties of the electrospun nanofibers were analyzed using a uniaxial testing machine (Instron 3365 dual column) under a cross-head speed of $5 \mathrm{~mm} /$ minute and gauge length of $25 \mathrm{~mm}$. From the stress-strain curves, tensile strength and elongation at break were calculated 


\subsection{Study of the release of Lavender oil components}

Agar gel circular slices were used to investigate the release of the lavender oil from the SA-PEO/LO nanofibers. The nanofibrous mats (disk with a diameter of $6 \mathrm{~mm}$ ) were placed in contact with the surface of the agar gel and the release was studied after $3,6,12,24$ and 48 hours at $1 \mathrm{~cm}$ distance from the centre of the mats. Five samples per time points were analyzed. SA-PEO nanofibers were used as reference samples for the same time points. Each sample was extracted with 0.1 $\mathrm{ml}$ of acetonitrile (ACN), sonicated for 10 minutes, and then centrifuged for 15 minutes at $7000 \times \mathrm{g}$. The supernatant was transferred into $0.25 \mathrm{ml}$ glass vials for analysis. The samples were analyzed by high-resolution liquid chromatographytandem mass spectrometry (LC-MS/MS) using an Acquity UPLC system coupled to a Synapt G2 qTOF mass spectrometer (Waters Inc, Milford MA, USA). All the chemicals used were purchased from Sigma Aldrich (Milano, Italy). Analyte separation was carried out on a T3 reversed phase column $(2.1 \mathrm{~mm} \times 100 \mathrm{~mm}$, Waters $)$ operated at a flow rate of 0.4 $\mathrm{ml} / \mathrm{min}$. Eluents were: A) water with $0.1 \%$ of formic acid, and B) ACN with $0.1 \%$ of formic acid. Analytes were separated using a linear gradient of eluent B in A (15 to $100 \%$ in 8 minutes) and detected in ESI+ mode. Compound identification was based on: matching the accurate mass and retention time with authentic lavender oil previously analyzed on the same system; matching the accurate masses, guessed brute formulas and tandem mass data of the compounds with the METLIN database. ${ }^{38}$ Three major lavender oil compounds were tracked in the release experiment: Linalool $(\mathrm{C} 10 \mathrm{H} 180$, detected as $[2 \mathrm{M}+\mathrm{Na}]^{+}$adduct at $\left.331,26 \mathrm{~m} / \mathrm{z}\right)$, Caryophillene $\left(\mathrm{C}_{15} \mathrm{H}_{24}\right.$, detected as $[\mathrm{M}+\mathrm{H}]^{+}$adduct at $\left.215.19 \mathrm{~m} / \mathrm{z}\right)$ and Caryophillene oxide $\left(\mathrm{C}_{15} \mathrm{H}_{24} \mathrm{O}\right.$, detected as $[\mathrm{M}+\mathrm{H} 2 \mathrm{O}+\mathrm{H}]^{+}$adduct at $203.18 \mathrm{~m} / \mathrm{z}$ ). The quantification of the detected species was obtained from the corresponding LC-MS chromatographic peak area.

\subsection{Antibacterial tests}

The antibacterial activity of the produced SA-PEO/LO nanofibers was tested against $S$. aureus. First, fibrous mats with a weight of $20 \mathrm{mg}$ were sterilized by UV irradiation inside a biohazard hood. For the antibacterial assays, the initial 108 CFUs $/ \mathrm{ml}$ inoculum of $S$. aureus was diluted to $10^{5}$ cells $/ \mathrm{ml}$, and $100 \mu \mathrm{L}$ of this solution were spread onto freshly prepared LB medium agar plates. The plates were placed in an incubator at $37{ }^{\circ} \mathrm{C}$ for 2 hours to allow a proper evaporation of the residual liquid medium. Then, the electrospun mats were positioned on the top of the solidified medium. After $24 \mathrm{~h}$ of incubation, photos of the plates were taken, and the inhibitory effect of LO was analyzed. SA-PEO mats without LO were used as control samples.

\subsection{In-vitro biocompatibility and anti-inflammatory studies}

\section{Cytotoxicity assay}

HFF-1 were grown in DMEM supplemented with $10 \% \mathrm{FBS}, 2$ $\mathrm{mmol} / \mathrm{I}$ L-glutamine, $100 \mathrm{IU} / \mathrm{ml}$ penicillin and $0.1 \mathrm{mg} / \mathrm{ml}$ streptomycin, in a humidified incubator at $37{ }^{\circ} \mathrm{C}$ with $5 \% \mathrm{CO}_{2}$.
Extraction medium from SA-PEO and SA-PEO/LO nanofibers was prepared adapting the procedure describing in ISO109935 standard test. Briefly, the nanofibers were sterilized under UV light for $30 \mathrm{~min}$. Then, the samples were immersed in the cell culture medium $\left(6 \mathrm{~cm}^{2}\right.$ of electrospun mat in $1 \mathrm{ml}$ of medium) for $24 \mathrm{~h}$ at $37^{\circ} \mathrm{C}$. HFF-1 cells were seeded in 96-well plates at a density of $8 \times 10^{3}$ cells per well in $100 \mu$ l of medium. After $24 \mathrm{~h}$ of culture, the medium was replaced with the extraction one, and the cells were incubated for further $24 \mathrm{~h}$. The viability of the cells, cultured with the fresh medium (control) and the extraction one, was determined measuring ATP levels by CellTiter-Glo assay (Promega Corporation Madison, WI, USA) according to manufacturer's protocol. This bioluminescence-based assay relies on the luciferase/luciferin reaction. The luminescence was recorded by Envision 2104 Multilabel Plate Reader (Perkin Elmer).

\section{Cytokine expression measurements}

HFF-1 cells were seeded at a density of $1.2 \times 10^{4}$ cells per $\mathrm{cm}^{2}$ and cultured for $24 \mathrm{~h}$. Then, the culture medium was removed and three different types of culture dishes were prepared: dishes containing fresh medium (control), or extraction medium from PEO, SA-PEO or SA-PEO/LO nanofibers. For each set of dishes, half of them were treated with $1 \mu \mathrm{g} / \mathrm{ml}$ LPS to induce inflammatory response. Culture medium with LPS and 1 $\mu \mathrm{M}$ of dexamethasone (a corticosteroid known to decrease inflammation) was used as a positive control in this procedure. After $6 \mathrm{~h}$, supernatants were collected and analyzed using ELISA to quantify the levels of IL- 6 and IL- 8 released into the medium, while cells were harvested and total RNA was extracted in order to quantify human IL- 6 and IL-8 expression by qRT-PCR.

\section{qRT-PCR cytokine mRNA quantification}

Total RNA of $1 \mu \mathrm{g}$ was reverse transcribed into first-strand cDNA by using SuperScript ${ }^{\circledR}$ VILO $^{\text {TM }}$ cDNA Synthesis Kit (Life Technologies, USA) in a final volume of $20 \mu$ l. HPRT-1 was used as the reference housekeeping gene in RT-PCR assays. Amplification of cytokines target genes and HPRT-1 was conducted with $50 \mathrm{ng}$ of cDNA in $20 \mu$ lof the reaction mixture by gene-specific primers using fluorogenic probes (TaqMan) and TaqMan ${ }^{\circledR}$ Universal PCR Master Mix, No AmpErase ${ }^{\circledR}$ UNG (Applied Biosystems). TaqMan primer/probes sets, spanning exon-exon junctions, for human IL-6 (Hs00985639_m1), IL-8 (Hs00174103_m1) and the housekeeping gene HPRT-1 (Hs02800695_m1) were used in PCR reactions. They were run in 96-well format on ViiA ${ }^{\mathrm{TM}} 7$ Real-Time PCR System (Applied Biosystems) using universal cycling conditions $\left(95^{\circ} \mathrm{C}, 10 \mathrm{~min}\right.$; $95{ }^{\circ} \mathrm{C}, 15 \mathrm{~s}$; and $60{ }^{\circ} \mathrm{C}, 1 \mathrm{~min}$ for 40 cycles). Finally, cycle threshold $(\mathrm{CT})$ values were determined by $\mathrm{ViiA}^{\mathrm{TM}} 7$ software v1.2.2.

\subsection{In-vivo studies on UVB-induced skin inflammation}

\section{Animal preparation}

Male C57BL/6J mice, 8 weeks old (Charles River, Calco, Italy), were used for in-vivo studies. Animals were group-housed in 
ventilated cages and had free access to food and water. They were maintained under a 12-hour light/dark cycle (lights on at $8: 00 \mathrm{am})$ at a controlled temperature of $\left(21 \pm 1^{\circ} \mathrm{C}\right)$ and relative humidity of $(55 \pm 10 \%)$. All experiments were carried out in accordance with the guidelines established by the European Communities Council Directive (Directive 2010/63/EU of 22 September 2010) and approved by the National Council on Animal Care of the Italian Ministry of Health. All efforts were made to minimize animal suffering and to use the minimal number of animals required to produce reliable results.

\section{UVB exposure model}

Animals were anaesthetized with a mixture of ketamine $(10 \%)$ and xylazine $(5 \%)$ administered in a single intraperitoneal injection. The dorsal skin was shaved, and mice were covered and orientated in order to have only the desired portion of skin (an area of approximately $1.5 \mathrm{~cm} 2$ ) exposed to a narrowband UVB light source that consisted of TL01 fluorescent tubes (Philips, UK, $\lambda \max =312 \mathrm{~nm}$ ) producing an even field of irradiation. The amount of UVB irradiation to which animals were exposed was calculated by using a calibrated meter (IL1400A with SEL240/UVB-1/TD filter, ABLE Instruments \& Controls Ltd, UK) at a distance of $15 \mathrm{~cm}$ from the tubes (equivalent to the mice distance). A maximal dose of $500 \mathrm{~mJ} / \mathrm{cm}^{2}$ was used for all experiments. After UVB irradiation, the exposed area was immediately covered with the SA-PEO or SA-PEO/LO nanofibers, or a commercial alginate product used as standard of care (3MTM TegadermTM Alginate). Then, the mice returned to their cages. Sham mice followed the same procedures without being exposed to UVB radiation.

\section{Cytokine expression measurements}

Mice were sacrificed at different time points, and skin samples from the UVB-exposed and non-exposed skin were removed and stored at $-80{ }^{\circ} \mathrm{C}$ until processing. Each sample was homogenized, subsequently centrifuged and the supernatant isolated and stored at $-80^{\circ} \mathrm{C}$. The expression of cytokines (IL-6, IL-1 $\beta$ and TNF- $\alpha$ ) was measured using ELISA quantikine kit (R\&D system), according to the manufacturer's instructions. The cytokine concentration was normalized against the total protein content for a given sample, as measured using the bicinchoninic acid (BCA) assay (Thermo Scientific, Rockford, IL, USA).

\subsection{Data analysis}

The amplification of the PCR product was expressed as the number of amplification cycles at which this particular product was first detected above the background (CT). The analysis of relative gene expression was performed by the comparative 2$\triangle \triangle C T$ method. The average $C T$ from three replicates was calculated for both target and HPRT-1 genes. The sample target quantity was corrected by the respective value of HPRT1. The $\triangle C T$ was determined to normalize for amounts of RNA used in reverse transcription reactions. The data are presented as fold change (2- $\triangle \Delta C T \pm$ S.E.M.). In animal studies, all data were presented as means \pm S.E.M.. For ELISA determination, the value obtained from each mouse was calculated as $\mathrm{pg} / \mathrm{mg}$ of protein. One-way ANOVA (for in vitro test) and Two way ANOVA (for in vivo test) were used to evaluate statistical significance, followed by Bonferroni's post-hoc test. GraphPad Prism 5 was used for all statistical analysis (GraphPad Software Inc. San Diego, CA, USA). P values less than 0.05 were considered significant.

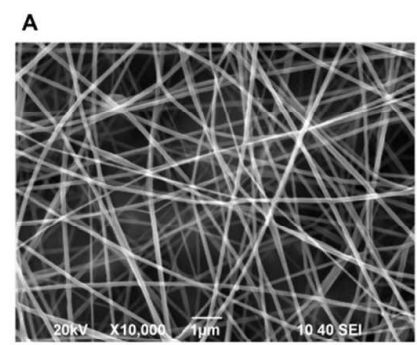

B

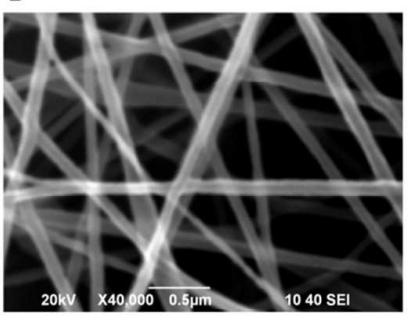

E

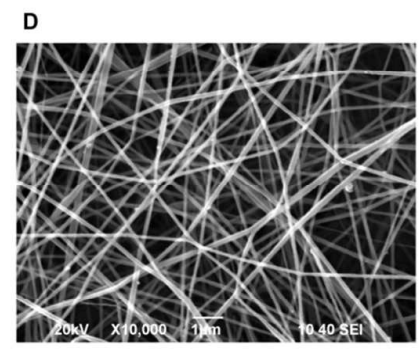

C

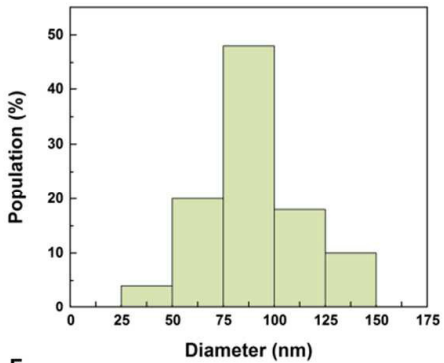

$\mathbf{F}$

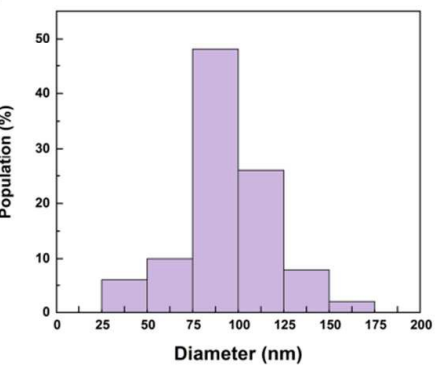

Figure 1. SEM images at different magnification and corresponding diameter distribution for SA-PEO $(A, B, C)$ and SA-PEO/LO (D, E, F) electrospun nanofibers, respectively. 


\section{Results and discussion}

\subsection{Physicochemical properties of the nanofibers}

In this study, PEO and Pluronic F127 were used to overcome the poor electrospinnability of the aqueous alginate solutions. ${ }^{10}$ The use of the surfactant Pluronic F127 facilitated also the emulsification of the essential oil in the aqueous phase, allowing the preparation of stable SA-PEO/LO solutions. This positively affected the morphology of the electrospun nanofibers. From Figure 1, where SEM images at different magnification of SA-PEO (Fig. $1 \mathrm{~A}$ and $1 \mathrm{~B}$ ) and SA-PEO/LO (Fig. $1 D$ and $1 E$ ) nanofibers are shown, it is possible to observe that the electrospun mats consisted of well-defined and bead-free nanofibers. An average diameter of $(91 \pm 21) \mathrm{nm}$ and $(93 \pm 22)$ $\mathrm{nm}$ was measured for SA-PEO (Fig. 1C) and SA-PEO/LO nanofibers (Fig. 1F), respectively. The image analysis revealed that the measured difference in diameter was not statistically significant $(p>0.05)$, indicating that the addition of the essential oil had a negligible effect on the morphology of the nanofibrous mats. In both cases, $85 \%$ of the fibers population exhibited a diameter in the range of 50-125 nm, with a most representative percentage $(48 \%)$ between $75-100 \mathrm{~nm}$.

After production, the alginate-based nanofibers were chemically analyzed in order to demonstrate the presence of LO. Figure 2 shows the Raman spectra of SA-PEO, SA-PEO/LO nanofibers, and pure LO. In the spectrum of SA-PEO fibrous mat (green curve in Fig. 2), the bands around 810, 880, 960, 1100 , and $1410 \mathrm{~cm}^{-1}$ are attributed to $\mathrm{CC}, \mathrm{CCH}, \mathrm{CO}, \mathrm{COC}$, and $\mathrm{COO}$ in the SA structure, respectively. ${ }^{39}$ In addition, the peaks at $840,1230,1280$, and $1490 \mathrm{~cm}^{-1}$ are assigned to the different vibrations of $\mathrm{CH}_{2}$ of both SA and PEO. ${ }^{39,40}$ The stretching of $\mathrm{CO}$ of PEO appears at $\sim 1070$ and $\sim 1140 \mathrm{~cm}^{-1} .40,41$ The spectrum of pure $\mathrm{LO}$ (black curve in Fig. 2) is characterized by the typical absorption bands of linalool $\left[\mathrm{C}=\mathrm{C}\right.$ of $\mathrm{RC}=\mathrm{C}\left(\mathrm{CH}_{3}\right)_{2}$ and $\mathrm{RC}=\mathrm{CH}_{2}$, respectively], detected at 1640 and $1670 \mathrm{~cm}^{-1}$. ${ }^{42}$ Moreover, the bands at 1452,1413 and $1378 \mathrm{~cm}^{-1}$ are attributed to $\mathrm{CH}_{3}$ and $\mathrm{CH}_{2}$; while, the peak at $1298 \mathrm{~cm}^{-1}$ is assigned to the $=\mathrm{CH}^{.33}$ The spectrum of the composite SA-PEO/LO fibers (violet curve in Fig. 2) shows the Raman peaks of all the constituent materials (SA, PEO, and LO), pointing out that the essential oil and particularly its active compounds were well incorporated into the fibers.

The wettability and the mechanical properties of the fibrous mats were investigated, because of their impact on the real use of the scaffolds for regenerative medicine. ${ }^{44}$ From contact angle measurements, we observed that both types of fibrous samples (SA-PEO and SA-PEO/LO) were highly hydrophilic, with an apparent WCA of $(21 \pm 2)^{\circ}$ to $(26 \pm 2)^{\circ}$, respectively. The contact angle was measured immediately after the positioning of the droplet on the sample surface, as the produced mats were able to absorb and uptake water. This property is useful for wound healing applications where hydrophilic or water soluble polymers are of interest for promoting the absorption of exudates and the delivery of water soluble drugs. ${ }^{45-47}$ The analysis of the mechanical properties of the SA-PEO and SAPEO/LO nanofibers revealed that they were characterized by a tensile strength of $(13 \pm 2) \mathrm{MPa}$ and $(9 \pm 1) \mathrm{MPa}$, and by an elongation at break of $(2.8 \pm 0.4) \%$ and $(1.6 \pm 0.2) \%$, respectively. Hence, the nanofibrous mats are robust enough to be easily handled without breaking, and they are flexible enough to adapt to skin wounds.

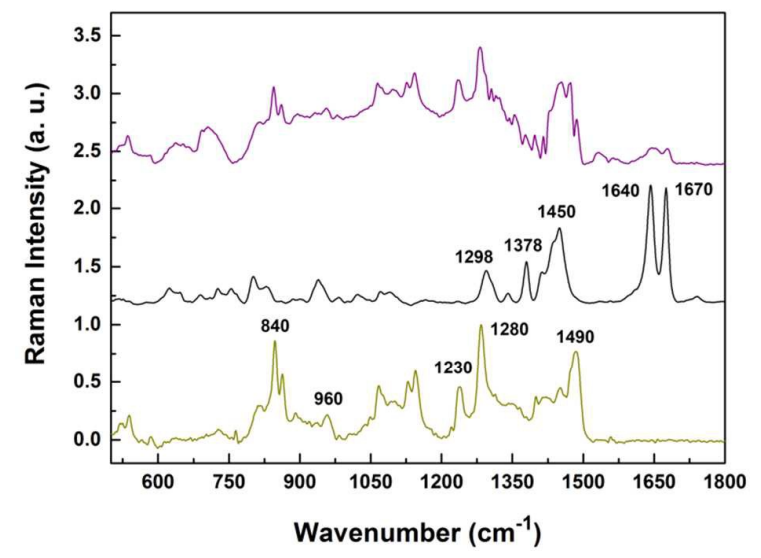

Figure 2. Comparison between the Raman spectra of the SA-PEO mats (green curve), pristine lavender essential oil (black curve), and composite SA-PEO/LO mats (violet curve).

\subsection{Release of LO components from the nanofibers}

The release profile of linalool from SA-PEO/LO nanofibers was investigated by high-resolution LC-MS/MS at different time points $(3,6,12,24$, and 48 hours), as shown in Figure 3. Together with linalool, caryophillene and caryophillene oxide, which are other characteristic components of lavender oil, were also analysed (Fig. S1). Agar gel was used as model system, in order to simulate a wet contact surface and to reproduce the same environment of the antibacterial tests (as in the following paragraph). We observed that linalool (Fig. 3) and the other components (Fig. S1) were efficiently released from the nanofibers, reaching a maximum after 6 hours. Then, the concentration decreases, as a consequence of the diffusion of the LO inside the agar gel. After 12 hours, the linalool concentration was comparable with that one at 3 hours, indicating that the nanofibrous mats still contained the essential oil. Even after 24 and 48 hours the amount of linalool released was appreciable. Therefore, the produced alginatebased nanofibers were active for more than 2 days, and able to release the antibacterial and anti-inflammatory agent. 


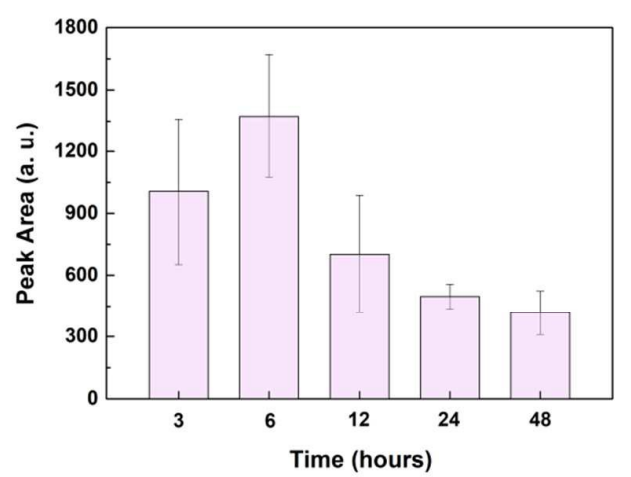

Figure 3. Release profile of linalool from SA-PEO/LO nanofibers at 3, 6, 12, 24, 48 hours.

\subsection{Antibacterial activity of the nanofibers}

Among the different types of microorganisms, Staphylococcus aureus is the most common bacterium that colonizes burn wounds in the first 48 hours after injury. ${ }^{48,}{ }^{49}$ Therefore, we selected $S$. aureus as model system to prove the antibacterial activity of the electrospun nanofibers in solid microbial cultures. From the analysis of SA-PEO mats (Figure 4A), we observed that they were readily colonized by $S$. aureus, and ineffective to block the bacteria growth. On the contrary, SA-PEO/LO nanofibers (Figure 4B) inhibited the proliferation of the microorganisms, and inhibition zones with an average diameter of $(21.7 \pm 1.6) \mathrm{mm}$ were visible in the LB medium agar plates. The obtained results are in agreement with a previous study of Edwards-Jones et al., where the antibacterial activity of pure LO against $S$. aureus has been demonstrated by detecting inhibition zones of approximately $20 \mathrm{~mm}$ in diameter. ${ }^{36}$ The main components of LO, linalool and linalyl acetate, are responsible for the antibacterial properties of this essential oil, ${ }^{50,51,52}$ with linalool being more effective than linalyl acetate. ${ }^{52}$ Furthermore, LO contains terpinen-4-ol that, like linalool, exhibits antimicrobial activity. ${ }^{50,51}$ Therefore, the various components of LO play an important role in making SA-PEO/LO effective in preventing infections, particularly in case of burn wounds.
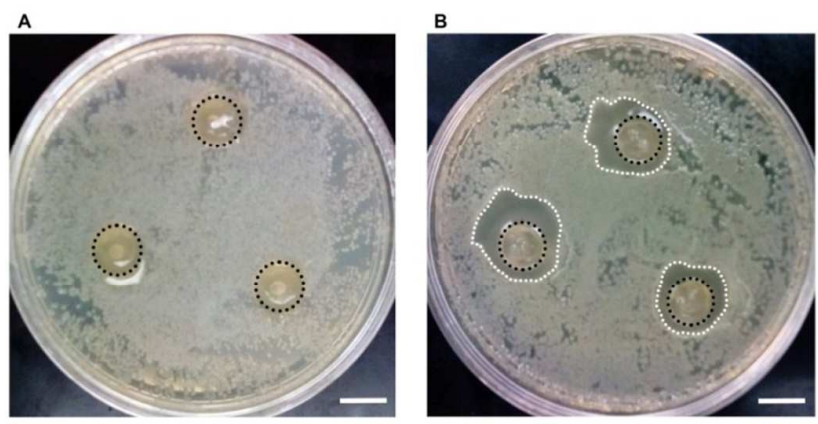

Figure 4. Photographs of the samples during the in-vitro antibacterial assay against $S$. aureus: (A) SA-PEO and (B) SA-PEO/LO nanofibers. The area of each sample is marked with a dotted black border, instead the inhibition zone with a dotted white border. Scale bar $=1 \mathrm{~cm}$.

\subsection{In-vitro biocompatibility assay}

Cytotoxicity assessment was performed in-vitro on HFF-1 cells in order to investigate the biocompatibility of SA-PEO nanofibers with and without LO. In Figure 5, the cell viability, measured as ATP content, is reported after 24 hours of exposure to nanofibers' extraction medium. It can be observed that $94 \%$ and $91 \%$ of the HFF-1 cells remained viable when incubated with SA-PEO and SA-PEO/LO extraction medium, respectively. No statistically significant differences $(p>0.05)$ were observed in the cell survival in comparison with the control, indicating no toxicity of SA-PEO and SA-PEO/LO fibrous samples. Therefore, the in-vitro results demonstrated that SAPEO/LO nanofibers were biocompatible without adverse reactions in human cell proliferation, in agreement with a previous study of Prashar et al. ${ }^{53}$

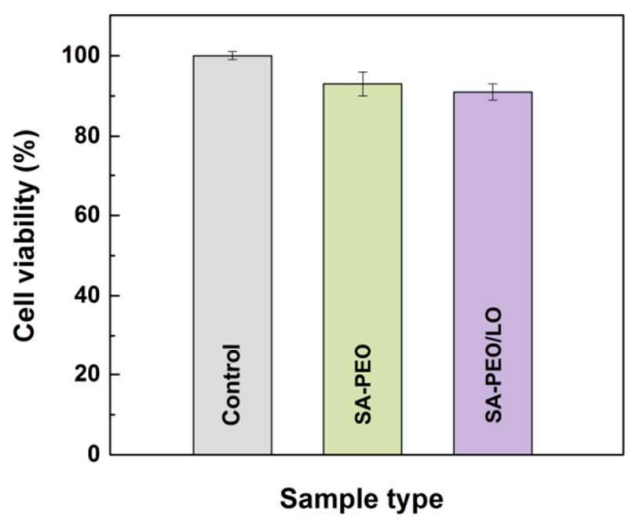

Figure 5. Viability of HFF-1 cells after $24 \mathrm{~h}$ of exposure to SA-PEO and SA-PEO/LO extraction medium. The control value is set to $100 \%$ and the values for the other conditions are normalized to it. Average percentage values \pm S.E.M. of three independent experiments, each performed in three technical replicates, are shown.

\subsection{In-vitro anti-inflammatory properties of the nanofibers}

In-vitro anti-inflammatory tests were conducted by stimulating the production of cytokines in HFF-1 cells with LPS. Then, the cell cultures were treated with DXM (positive control) or extraction media obtained from PEO, SA-PEO and SA-PEO/LO nanofibers, and the induction (relative mRNA levels) and release (protein expression levels) of two pro-inflammatory cytokines mainly involved in the inflammatory wound process, human IL-6 and human IL-8 were investigated (Figure 6, S2 and Table 1). As expected, we observed a reduction of $93 \%$ (Figure $6 \mathrm{~A}$ ) and $81 \%$ (Figure $6 \mathrm{~B}$ ) of the mRNA levels of IL-6 and IL-8 for cells treated with DXM, respectively. In fact, DXM is conventionally used as anti-inflammatory drug. If pure PEO nanofibers had no effect in reducing mRNA levels for both cytokines (Fig. S2), the analysis of the electrospun samples revealed that SA-PEO nanofibers, on the other hand, possessed anti-inflammatory activity. Indeed, they effectively reduced the mRNA levels of about 50\% for both IL-6 and IL-8. 


\section{Journal of Materials Chemistry B}

This effect was accentuated by the addition of lavender oil. In fact, HFF-1 cells incubated with SA-PEO/LO extraction medium presented a reduction of $75 \%$ and $68 \%$ of IL- 6 and IL- 8 mRNA expression $(p<0.001)$, respectively. Furthermore, as shown in Figures $6 \mathrm{C}, 6 \mathrm{D}$ and Table 1 , a reduction of the release of IL- 6 and IL- 8 was also observed in the cell culture supernatants after LPS stimulation and treatment with the alginate-based extraction media. remedy for inflammation-related symptoms, as shown also by Kang et al. for the seaweed Sargassum fulvellum. ${ }^{57}$
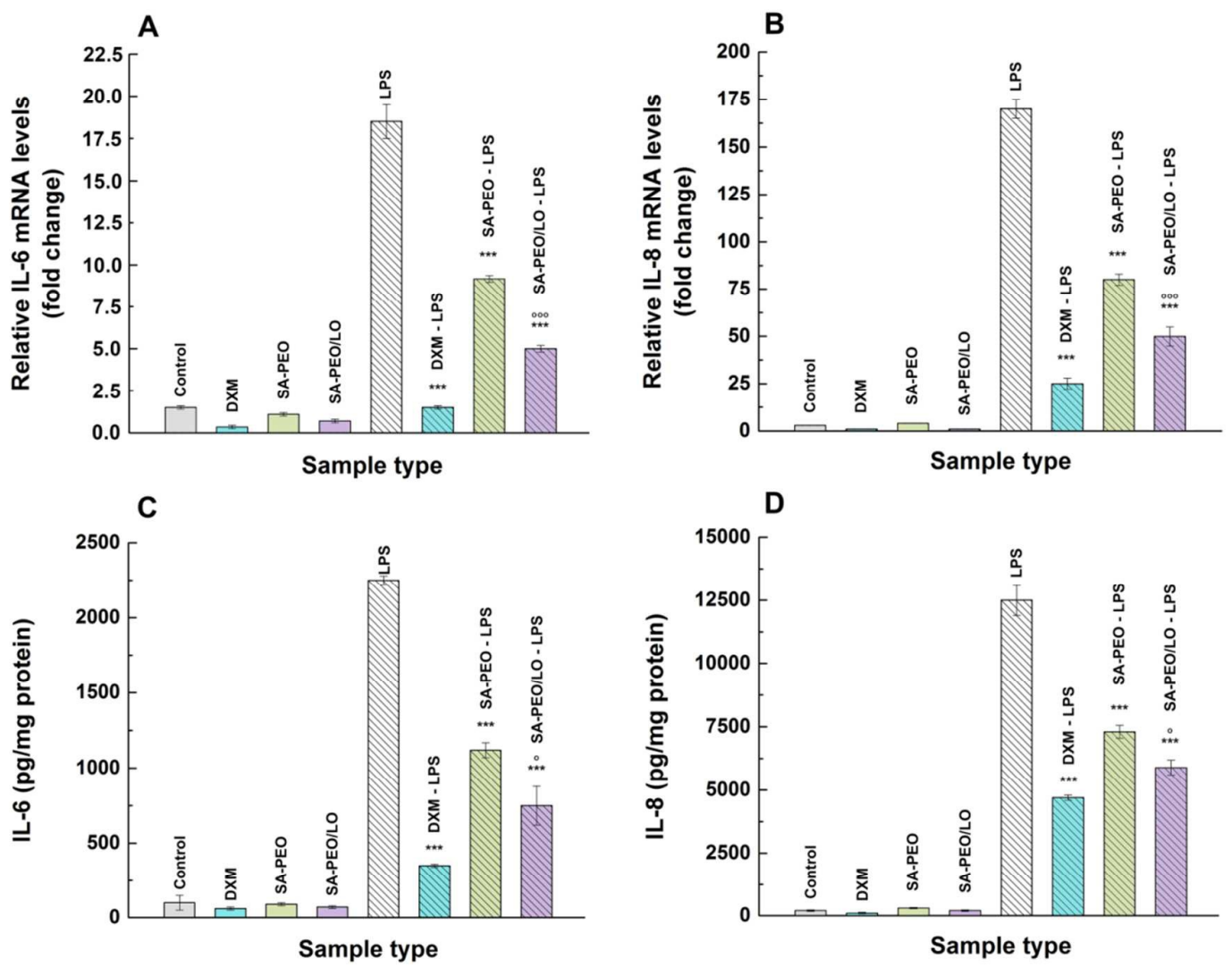

Figure 6. Anti-inflammatory effect of SA-PEO and SA-PEO/LO nanofibers, evaluated on HFF-1 cells stimulated with LPS. Histograms show the mean values of mRNA levels of (A) IL-6 and (B) IL-8, and the amount of (C) IL- 6 and (D) IL-8 after ELISA evaluation. The first 4 bars in each panel are the control samples. Data are expressed as average \pm S.E.M. ( ${ }^{* * *} p<0.001$ vs. LPS group; ${ }^{\circ} p<0.05,{ }^{\circ 00} p<0.001$ vs. alginate group). The graphs are representative of three independent experiments, each performed in three technical replicates.

In particular, SA-PEO/LO nanofibers induced a statistically significant decrease $(\mathrm{p}<0.05)$ of IL-6 $(66 \%)$ and IL-8 $(49 \%)$ concentrations in comparison with SA-PEO samples $(51 \%$ for IL-6, and $43 \%$ for IL-8).

It is known from the literature that $\mathrm{Ca}^{2+}$ ions from Ca-alginate systems, ${ }^{22}$ and oligosaccharides derived from polysaccharides ( $\beta$-glucan, xylogucan, chitin, pectin, $\alpha$-L-guluronate, and $\beta$-Dmannuronate) can stimulate human cells to produce cytokines. $^{54,55}$ Although guluronate and mannuronate oligomers are pro-inflammatory agents, polyguluronate and polymannuronate, which constitute sodium alginate, are not active in cytokine induction. ${ }^{54,56}$ On the contrary, seaweeds that are rich in sodium alginate are traditionally used as
Table 1: In-vitro anti-inflammatory effect of SA-PEO and SA-PEO/LO nanofibers. The percentages of reduction for mRNA expression and protein release detected in the supernatants (IL-6 and IL-8) are reported. Average percentage values \pm S.E.M. of three independent experiments, each performed in three technical replicates, are shown.

\begin{tabular}{|c|c|c|c|c|}
\hline & \multicolumn{2}{|c|}{ IL-6 } & \multicolumn{2}{c|}{ IL-8 } \\
\hline Sample & $\begin{array}{c}\text { mRNA } \\
\text { levels (\%) }\end{array}$ & $\begin{array}{c}\text { Protein } \\
\text { levels (\%) }\end{array}$ & $\begin{array}{c}\text { mRNA } \\
\text { levels (\%) }\end{array}$ & $\begin{array}{c}\text { Protein } \\
\text { levels (\%) }\end{array}$ \\
\hline DXM & $93 \pm 2$ & $83 \pm 1$ & $81 \pm 4$ & $64 \pm 3$ \\
\hline SA-PEO & $51 \pm 4$ & $51 \pm 4$ & $50 \pm 4$ & $43 \pm 1$ \\
\hline SA-PEO/LO & $75 \pm 4$ & $66 \pm 2$ & $68 \pm 5$ & $49 \pm 4$ \\
\hline
\end{tabular}


Furthermore, the anti-inflammatory activity of lavender essential oil has been reported in several studies. ${ }^{32,33,58-60}$. It has been demonstrated that linalool and linalyl acetate, the inflammation through different pathways. ${ }^{58}$ Linalool significantly reduces the production of IL- 6 both in-vitro and in-vivo through the mitogen-activated protein kinase (MAPK) pathway that in turn regulates the expression of inflammatory enzymes and cytokines. ${ }^{32}$ In this way, linalool can inhibit the phospho-p38 MAPK, phospho-ERK, and phospho-JNK in LPSstimulated cells. ${ }^{32}$ Moreover, the anti-inflammatory activity of linalool can be mainly due to the inducible nitric oxide synthase (iNOS) activity that controls other inflammatory factors, such as nitric oxide (NO), prostaglandins (PG) and cyclooxygenase. $^{61}$ Linalyl acetate shows, instead, strong lipoxygenase inhibitory effects that are mediated through the lipoxygenase pathway associated to the inflammation. ${ }^{62}$

\subsection{In-vivo application of the alginate-based dressings}

Redness and erythema, due to the vasodilation of the cutaneous blood vessels, are typical cutaneous manifestations of the response of the skin to UVB-induced inflammation, ${ }^{27,63}$ and they are characterized by the up-regulation of proinflammatory cytokines, together with the release of neuropeptides, histamine, prostaglandins, serotonin and oxygen radicals. ${ }^{26}$ Erythema is a multifactorial and complicated reaction in which NO, PG, and cytokines play important roles. ${ }^{27}$ In our study, the exposure of the animal skin to UVB irradiation produced evident erythema with signs of mild burn that lead to visible skin lesions and scar formation in 48-96 hours (Figure $7 A)$. Redness became apparent after 12-24 hours from the UVB-induced inflammation, but none of the animals developed blisters. We observed a more evident skin injury after 48 hours from the exposure. The inflammation response was analyzed by detecting the cutaneous levels of cytokines (IL-6, IL-1 $\beta$, TNF$\alpha)$ at different time-points (from 6 to 96 hours) after the acute UVB exposure (photographs of the animals at time point 0 are showed in Fig. S3). A significant increment of the produced cytokines was observed for the animals exposed to UVB light in comparison with control animals (not UVB-exposed). For the latter, the levels of IL- 6 and IL- $1 \beta$ were extremely low, and TNF- $\alpha$ level was almost below the detection limit. For animals with an untreated burn wound, the time-course of cytokines expression revealed that IL-6, IL-1 $\beta$ and TNF- $\alpha$ gradually increased after 24 hours from the injury, exhibiting a maximum at 48 hours (Table S1-S3), as shown in Figure 7B, 7C and 7D. After 96 hours, all the cytokine levels significantly decreased but they were still quite high. Our data correlate very well with human skin, where UVB irradiation alters the immune function and migration of Langerhans cells and dermal dendritic cells and these cells produce high levels of TNF- $\alpha$, IL-1 $\beta$, IL- 6 and IL- $8 .{ }^{64}$

On the contrary, when the burns were treated with the SAPEO or SA-PEO/LO nanofibrous dressings, no evident burn marks were visible on the skin of the animals already after 24 hours from the injury, and the erythema completely disappeared within 48 hours (Figure 7A). The photo-damage recovery was confirmed by the down-regulation of the three cytokines (Figure 7B, 7C, 7D, and Table S1-S3). Specifically, after 24 hours the levels of IL-6, IL-1 $\beta$, and TNF- $\alpha$ were up to 4 , 10 , and 7 times lower than for the UVB-irradiated group of animals (not treated with the dressing), respectively. The antiinflammatory effect of the nanofibers was even more evident after 48 hours, when the levels of IL- 6, IL- $1 \beta$, and TNF- $\alpha$ for the treated animals were up to 7,24 , and 19 times lower than those for the untreated group, respectively. After 96 hours, cytokine levels returned to the control values. The in-vivo results on cytokine profile after UVB irradiation were consistent with the in-vitro data. Remarkably, SA-PEO nanofibers played a fundamental role in the suppression of cytokines production and their anti-inflammatory activity was successfully combined with that one of the lavender essential oil. The absence of erythema for the animal groups treated with the alginate-based nanofibers demonstrated that UVBinduced inflammation reaction was controlled and well prevented, therefore, the electrospun dressings were proven appropriate for the management of burn wounds.

The UVB burn in-vivo model was tested also on a commercially available alginate-based dressing (Tegaderm ${ }^{\mathrm{TM}}$ ) and the obtained results are shown in Tables S1-S3. We noticed that the inhibitory effect of the electrospun nanofibers on cytokine production was higher than that one of Tegaderm ${ }^{\mathrm{TM}}$ after the first 6 hours from the UVB exposure; the results were comparable for the other time points. One of the other advantages of the electrospun nanofibers with respect to Tegaderm is their high conformability to the wound site. They are, in fact, able to adapt to the injured tissue, offering protection against thermal and mechanical stresses. Moreover, they worked as solid support for the delivery of LO to the burn.

\section{Conclusions}

In conclusion, we demonstrated that nanofibrous dressings of sodium alginate and lavender essential oil not only possessed antibacterial activity against $\mathrm{S}$. aureus but they also effectively inhibited the production of pro-inflammatory cytokines both in-vitro and in-vivo. This resulted in a fast recovery of animals exposed to UVB irradiation, without the appearance of erythema on their injured skin. We can state that in the produced electrospun dressings both the selected materials had an active effect in promoting the healing of the burn. The strong anti-inflammatory action of sodium alginate was evident in all the conducted investigations. On the other hand, lavender oil expressed a high antimicrobial effectiveness and also acted to control the induced inflammation. Further studies are needed to better clarify the potentiality of these dressings on deep burns and other types of wounds and skin damages. However, we believe that the properties of the here proposed natural dressings are attractive for the future generations of wound care systems. 
A
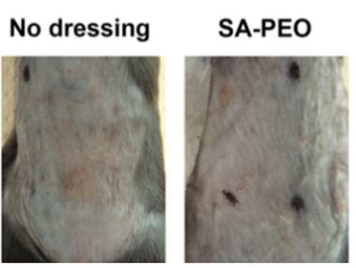

$48 \mathrm{~h}$
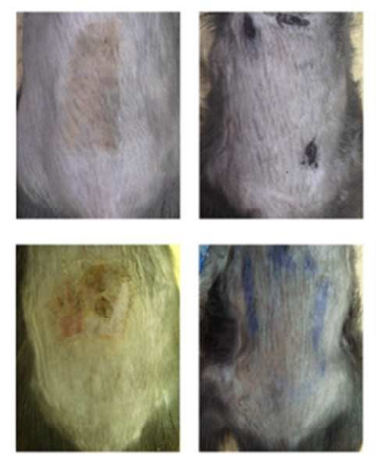

SA-PEO/LO

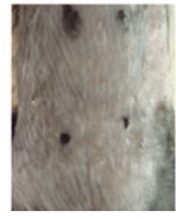

$96 \mathrm{~h}$
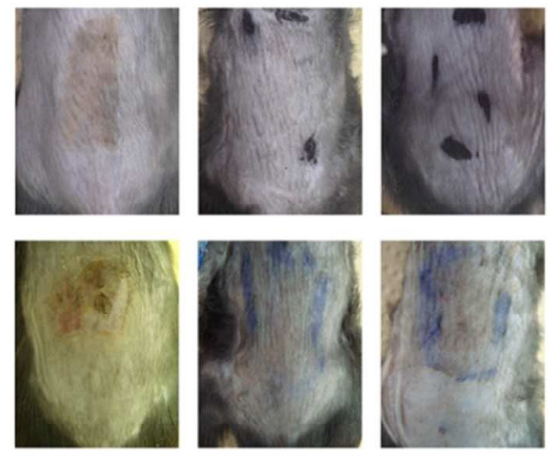

B

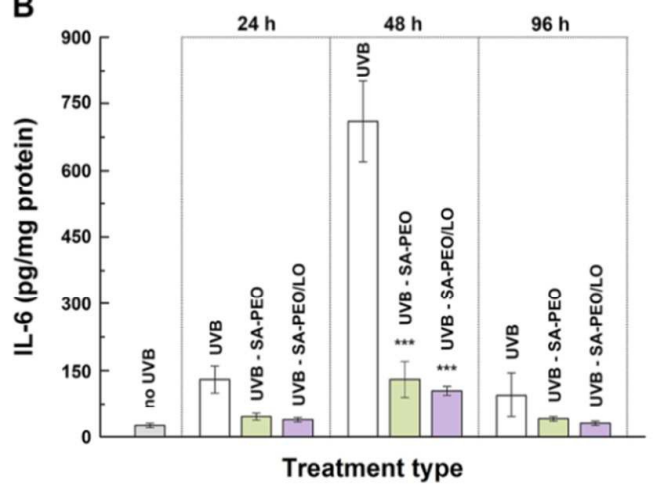

C
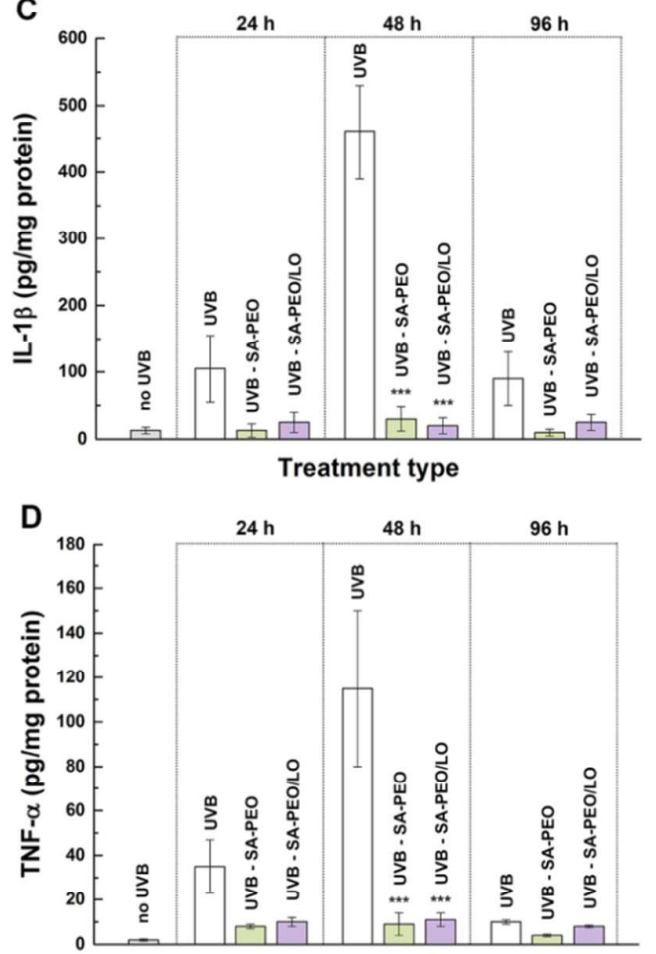

Treatment type

Figure 7. (A) Representative photographs of the skin of UVB exposed mice at three different time points (24 h, $48 \mathrm{~h}$, and $96 \mathrm{~h})$ : untreated (no dressing), and treated with the alginate-based dressings without (SA-PEO) and with lavender oil (SA-PEO/LO). Time course of the effects of UVB induced inflammation on cytokine expression: (B) IL-6 (a), (C) IL-1 1 , (D) TNF- $\alpha$. Each bar is the average \pm SD of five experiments (a group of five animals) after 24,48 and 96 hours of injury. ${ }^{* * *} p<0.001$ vs. UVB irradiated group. 


\section{References}

1.

2. M. Abrigo, S. L. McArthur and P. Kingshott, Macromolecular Bioscience, 2014, 14, 772-792.

3. N. Mayet, Y. E. Choonara, P. Kumar, L. K. Tomar, C. Tyagi, L. C. Du Toit and V. Pillay, Journal of Pharmaceutical Sciences, 2014, 103, 2211-2230.

4. U.S. Patent 5,256,477, 1993.

5. P. Zahedi, I. Rezaeian, S.-O. Ranaei-Siadat, S.-H. Jafari and P. Supaphol, Polymers for Advanced Technologies, 2010, 21, 77-95.

6. L. I. Moura, A. M. Dias, E. Carvalho and H. C. de Sousa, Acta biomaterialia, 2013, 9, 7093-7114.

7. W. Paul and C. P. Sharma, Trends in Biomaterials and Artificial Organs, 2004, 18.

8. K. Y. Lee and D. J. Mooney, Progress in Polymer Science, 2012, 37, 106-126.

9. H. Hajiali, J. A. Heredia-Guerrero, I. Liakos, A. Athanassiou and E. Mele, Biomacromolecules, 2015, 16, 936-943.

10. C. A. Bonino, K. Efimenko, S. I. Jeong, M. D. Krebs, E. Alsberg and S. A. Khan, Small, 2012, 8, 1928-1936.

11. J.-W. Lu, Y.-L. Zhu, Z.-X. Guo, P. Hu and J. Yu, Polymer, 2006, 47, 8026-8031.

12. Y. Zhang, C. T. Lim, S. Ramakrishna and Z. M. Huang, Journal of materials science. Materials in medicine, 2005, 16, 933-946.

13.

G. D. Mogosanu and A. M. Grumezescu, International journal of pharmaceutics, 2014, 463, 127-136.

14. Y. Qin, Polymer International, 2008, 57, 171-180.

15. B. Kneafsey, M. O'Shaughnessy and K. C. Condon, Burns, 1996, 22, 40-43.

16. M. D. Dantas, D. R. Cavalcante, F. E. Araujo, S. R. Barretto, G. T. Aciole, A. L. Pinheiro, M. A. Ribeiro, I. B. Lima-Verde, C. M. Melo, J. C. Cardoso and R. L. Albuquerque Junior, Journal of photochemistry and photobiology. B, Biology, 2011, 105, 51-59.

$17 . \quad$ L. K. Branski, A. Al-Mousawi, H. Rivero, M. G. Jeschke, A. P. Sanford and D. N. Herndon, Surgical infections, 2009, 10, 389-397.

18. I. Brook and J. G. Randolph, Journal of Trauma and Acute Care Surgery, 1981, 21, 313-318.

19. S. Opasanon, P. Muangman and N. Namviriyachote, International Wound Journal, 2010, 7, 467-471.

20. J. G. Thomas, W. Slone, S. Linton, T. Okel, L. Corum and S. L. Percival, Journal of wound care, 2011, 20, 124,126-128.

21. E. Öztürk, C. Ağalar, K. Keçeci and E. B. Denkbaş, Journal of Applied Polymer Science, 2006, 101, 1602-1609.

22. G. Chan and D. J. Mooney, Acta biomaterialia, 2013, 9, 9281-9291.

23. A. Thomas, K. G. Harding and K. Moore, Biomaterials, 2000, 21, 1797-1802.

24. K. C. Chang, H. Ma, W. C. Liao, C. K. Lee, C. Y. Lin and C. C. Chen, Burns, 2010, 36, 1059-1066.

25. L. H. Evers, D. Bhavsar and P. Mailander, Experimenta dermatology, 2010, 19, 777-783.

26.

N. E. Saade, I. W. Nasr, C. A. Massaad, B. SafiehGarabedian, S. J. Jabbur and S. A. Kanaan, British journal of pharmacology, 2000, 131, 1317-1324.

27.

G. J. Clydesdale, G. W. Dandie and H. K. Muller, Immunology and cell biology, 2001, 79, 547-568.
28. H. M. A. Cavanagh and J. M. Wilkinson, Phytotherapy Research, 2002, 16, 301-308.

29. L. Cuttle, J. Pearn, J. R. McMillan and R. M. Kimble, Burns, 2009, 35, 768-775.

30. L. R. Williams, J. K. Stockley, W. Yan and V. N. Home, International Journal of Aromatherapy, 1998, 8, 30-40.

31. R. Shellie, L. Mondello, P. Marriott and G. Dugo, Journal of chromatography. A, 2002, 970, 225-234.

32. M. Huo, X. Cui, J. Xue, G. Chi, R. Gao, X. Deng, S. Guan, J. Wei, L. W. Soromou, H. Feng and D. Wang, Journal of Surgical Research, 2013, 180, e47-e54.

33. A. T. Peana, P. S. D'Aquila, F. Panin, G. Serra, P. Pippia and M. D. L. Moretti, Phytomedicine, 2002, 9, 721-726.

34. F. Bakkali, S. Averbeck, D. Averbeck and M. Idaomar, Food and Chemical Toxicology, 2008, 46, 446-475.

35. I. Liakos, L. Rizzello, D. J. Scurr, P. P. Pompa, I. S. Bayer and A. Athanassiou, International journal of pharmaceutics, 2014, 463, 137-145.

36. V. Edwards-Jones, R. Buck, S. G. Shawcross, M. M. Dawson and K. Dunn, Burns, 2004, 30, 772-777.

37. K. Balasubramanian and K. M. Kodam, RSC Advances, 2014, 4, 54892-54901.

38. R. Tautenhahn, K. Cho, W. Uritboonthai, Z. Zhu, G. J. Patti and G. Siuzdak, Nature biotechnology, 2012, 30, 826-828.

39. N. P. Ivleva, M. Wagner, H. Horn, R. Niessner and C. Haisch, Analytical and bioanalytical chemistry, 2009, 393, 197-206.

40. K. Pielichowska, S. Głowinkowski, J. Lekki, D. Biniaś, K. Pielichowski and J. Jenczyk, European Polymer Journal, 2008, 44, 3344-3360.

41. T. Yoshihara, H. Tadokoro and S. Murahashi, The Journa of Chemical Physics, 1964, 41, 2902-2911.

42. D. J. Daferera, P. A. Tarantilis and M. G. Polissiou, Journal of Agricultural and Food Chemistry, 2002, 50, 5503-5507.

43. P. Jentzsch, L. Ramos and V. Ciobotă, Cosmetics, 2015, 2, 162.

44. B. Cortese, M. O. Riehle, S. D'Amone and G. Gigli, Journal of Colloid and Interface Science, 2013, 394, 582-589.

45. K. Kataria, A. Gupta, G. Rath, R. B. Mathur and S. R. Dhakate, International journal of pharmaceutics, 2014, 469, 102-110.

46. N. Mohamad, M. C. I. Mohd Amin, M. Pandey, N. Ahmad and N. F. Rajab, Carbohydrate Polymers, 2014, 114, 312 320.

47. Y. Zhou, D. Yang, X. Chen, Q. Xu, F. Lu and J. Nie, Biomacromolecules, 2008, 9, 349-354.

48. C. Childs, V. Edwards-Jones, D. M. Heathcote, M. Dawson and P. J. Davenport, Burns, 1994, 20, 514-521.

49. D. Church, S. Elsayed, O. Reid, B. Winston and R. Lindsay, Clinical Microbiology Reviews, 2006, 19, 403-434

50. R. Prusinowska and B. Śmigielski Krzysztof, Journal, 2014 60, 56.

51. S. Inouye, T. Takizawa and H. Yamaguchi, Journal of Antimicrobial Chemotherapy, 2001, 47, 565-573.

52. M. Sokovic, P. D. Marin, D. Brkic and L. J. L. D. V. Griensven, Food, 2008, 1, 220-226.

53. A. Prashar, I. C. Locke and C. S. Evans, Cell proliferation, 2004, 37, 221-229.

54. M. Iwamoto, M. Kurachi, T. Nakashima, D. Kim, K. Yamaguchi, T. Oda, Y. Iwamoto and T. Muramatsu, FEBS letters, 2005, 579, 4423-4429. 


\section{Journal of Materials Chemistry B}

55. C. A. Ryan and E. E. Farmer, Annual Review of Plant Physiology and Plant Molecular Biology, 1991, 42, 651674.

56. Y. Iwamoto, X. Xu, T. Tamura, T. Oda and T. Muramatsu, Bioscience, biotechnology, and biochemistry, 2003, 67, 258-263.

57. J. Y. Kang, M. N. Khan, N. H. Park, J. Y. Cho, M. C. Lee, H. Fujii and Y. K. Hong, Journal of ethnopharmacology, 2008, 116, 187-190.

58. M. G. Miguel, Molecules (Basel, Switzerland), 2010, 15, 9252-9287.

59. J. S. Raut and S. M. Karuppayil, Industrial Crops and Products, 2014, 62, 250-264.

60. T. Ueno-lio, M. Shibakura, K. Yokota, M. Aoe, T. Hyoda, R. Shinohata, A. Kanehiro, M. Tanimoto and M. Kataoka, Life sciences, 2014, 108, 109-115.

61. A. T. Peana, S. Marzocco, A. Popolo and A. Pinto, Life sciences, 2006, 78, 719-723.

62. A. Wei and T. Shibamoto, J Agric Food Chem, 2010, 58, 7218-7225.

63. G. Logan and D. L. Wilhelm, Nature, 1963, 198, 968-969.

64. N. Bechetoille, C. Dezutter-Dambuyant, O. Damour, V. Andre, I. Orly and E. Perrier, Tissue engineering, 2007, 13, 2667-2679. 